\title{
Enhanced Stability of Single-Layer w-Gallenene through Hydrogenation
}

\author{
S. V. Badalov, ${ }^{* \dagger}$ M. Yagmurcukardes, ${ }^{*}, \S$ F. M. Peeters, ${ }^{\S}$ and H. Sahin ${ }^{\dagger, \ddagger(0)}$ \\ ${ }^{\dagger}$ Department of Photonics and ${ }^{\ddagger}$ ICTP-ECAR Eurasian Center for Advanced Research, Izmir Institute of Technology, 35430 Izmir, \\ Turkey \\ ${ }^{\S}$ Department of Physics, University of Antwerp, Groenenborgerlaan 171, B-2020 Antwerp, Belgium
}

Supporting Information

ABSTRACT: Using density functional theory based first-principles calculations, the effect of surface hydrogenation on the structural, dynamical, electronic, and mechanical properties of monolayer washboard-gallenene (w-gallenene) is investigated. It is found that the dynamically stabilized strained monolayer of w-gallenene has a metallic nonmagnetic ground state. Both one-sided and two-sided hydrogenations of w-gallenene suppress its dynamical instability even when unstrained. Unlike one-sided hydrogenated monolayer w-gallenene (os-w-gallenene), two-sided hydrogenated monolayer $\mathrm{w}$-gallenene (ts-w-gallenene) possesses the same crystal structure as w-gallenene. Electronic band structure calculations reveal that monolayers of hydrogenated derivatives of $\mathrm{w}$-gallenene exhibit also metallic nonmagnetic ground state. Moreover, the linear-elastic
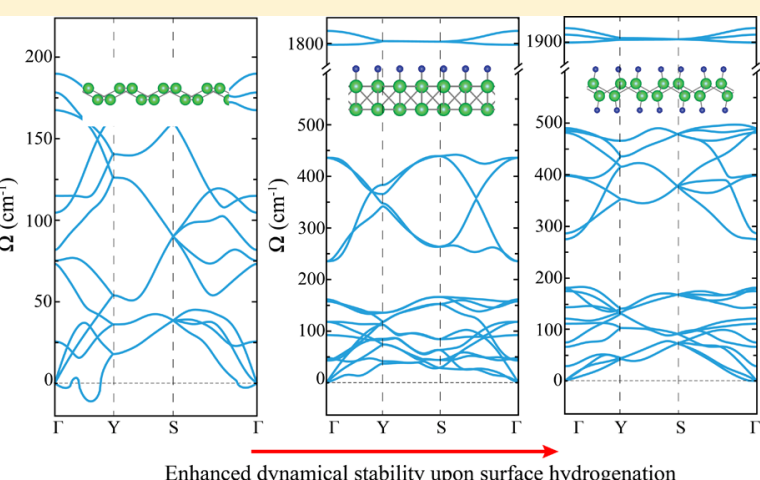
constants, in-plane stiffness and Poisson ratio, are enhanced by hydrogenation, which is opposite to the behavior of other hydrogenated monolayer crystals. Furthermore, monolayer wgallenene and ts-w-gallenene remain dynamically stable up to relatively higher biaxial strains as compared to borophene. With its enhanced dynamical stability, robust metallic character, and enhanced linear-elastic properties, hydrogenated monolayer wgallenene is a potential candidate for nanodevice applications as a two-dimensional flexible metal.

\section{INTRODUCTION}

Since the successful isolation of graphene ${ }^{1,2}$ from its bulk counterpart, graphite, two-dimensional (2D) materials have received immense interest in material science and device technology owing to their unconventional properties. ${ }^{3-6}$ It has stimulated researchers to synthesize other monolayer monoatomic crystals such as silicene, ${ }^{7-9}$ germanene, ${ }^{10-13}$ stanene, ${ }^{14,15}$ phosphorene, ${ }^{16}$ and borophene, ${ }^{17}$ which exhibit a wide range of electronic, thermal, mechanical, and chemical properties, making them promising candidates for many different applications.

Gallium is an enchanting metallic element because it favors covalency over metallicity in its bulk form. ${ }^{18}$ Therefore, gallium possesses one of the most diverse phase diagrams of the metallic elements, and it is a nontoxic liquid metal at room temperature. ${ }^{19,20}$ It displays various types of phases such as a boron-like molecular and close-packed metallic phase under nonstandard pressure and temperature. ${ }^{19,21,22}$ Furthermore, the extreme covalent character of the $\mathrm{Ga}_{2}$ pairs results in a dimer-like behavior. Hence, among the monoatomic materials, $\alpha$-Ga is the only one that exhibits both metallic and molecular character at zero pressure. ${ }^{19}$ On the other hand, gallium is known to form the basis of the following semiconductors: $\mathrm{GaX}$ $(\mathrm{X}=\mathrm{N}, \mathrm{P}, \mathrm{As})$, which are used primarily in electronic circuit and semiconductor devices. ${ }^{20,23,24}$ Zhang et al. reported that epitaxially grown $\mathrm{Ga}$ films on the wide-band-gap semiconductor $\mathrm{GaN}(0001)$ demonstrate a novel platform for exploring atomic-scale $2 \mathrm{D}$ superconductors, which is important for understanding interface superconductivity. ${ }^{25}$ Zhou et al. showed that monolayer GaSe possesses the strongest secondharmonic generation intensity among similar 2D crystals. $^{26}$ Moreover, 2D GaS and $\mathrm{GaO}$ layers with exciting functional properties were also successfully synthesized. ${ }^{27}$ In our recent study, we reported that monolayers of Ga-monochalcogenides $(\mathrm{GaS}$ and $\mathrm{GaSe})$ are ductile and flexible materials. ${ }^{28}$

Apart from its binary compounds, monoatomic crystals of gallium in the $2 \mathrm{D}$ limit have not been studied in detail up to date. Xing et al. reported that the quantum Griffiths singularity is observed experimentally in atomically thin gallium films by showing a superconductor-metal transition with increasing magnetic field. ${ }^{29}$ Very recently, Kochat et al. reported the successful synthesis of two-dimensional atomically thin "gallenene" phases on various substrates. ${ }^{30}$ They also showed that free-standing gallenene can be dynamically stabilized upon the application of biaxial strain.

Received: July 30, 2018

Revised: November 4, 2018

Published: November 12, 2018 

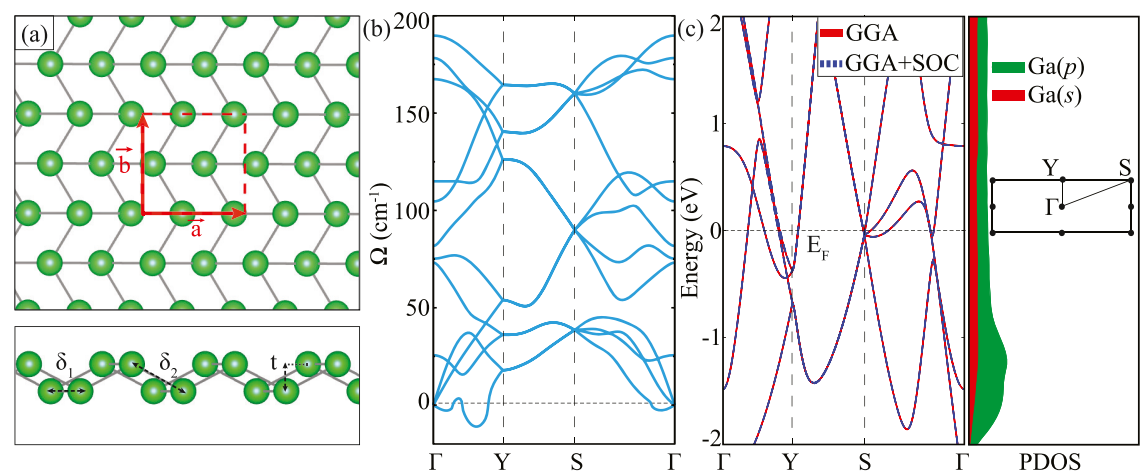

Figure 1. (a) Top and side views of monolayer w-gallenene. Red dashed lines represent the rectangular unit cell; $\vec{a}$ and $\vec{b}$ are the lattice vectors. (b) The phonon band diagram of w-gallenene. (c) The electronic band diagram and orbital projected partial density of states (PDOS) of w-gallenene. Inset represents the high-symmetry points in the first $\mathrm{BZ}$ of the orthorhombic reciprocal unit cell. The Fermi level is set to zero.

Table 1. Calculated Parameters for Monolayers of Bare w-Gallenene and Its Hydrogenated Derivatives ${ }^{a}$

$\begin{array}{lccccccccccccc} & a(\AA) & b(\AA) & \delta_{1}(\AA) & \delta_{2}(\AA) & t(\AA) & \Delta \rho_{\mathrm{Ga}-\mathrm{H}}\left(\mathrm{e}^{-}\right) & \mu\left(\mu_{\mathrm{B}}\right) & E_{\mathrm{Coh} / \mathrm{atom}}(\mathrm{eV}) & \Phi(\mathrm{eV}) & C_{\mathrm{a}}(\mathrm{N} / \mathrm{m}) & C_{\mathrm{b}}(\mathrm{N} / \mathrm{m}) & \nu_{\mathrm{a}} & \nu_{\mathrm{b}} \\ \text { bare } & 4.65 & 4.82 & 2.71 & 2.73 & 1.27 & & 0 & 2.32 & 4.03 & 25 & 25 & 0.20 & 0.20 \\ \text { os } & 4.65 & 4.65 & 2.82 & 2.90 & 3.70 & 0.2 & 0 & 2.25 & 5.17 & 52 & 52 & 0.26 & 0.26 \\ \text { ts } & 4.62 & 4.55 & 2.72 & 2.66 & 4.44 & 0.3 & 0 & 2.25 & 5.22 & 48 & 47 & 0.25 & 0.25\end{array}$

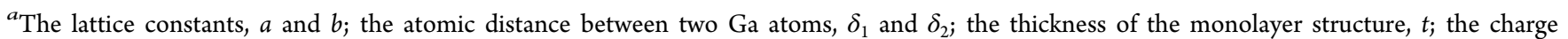
donation from Ga to $\mathrm{H}, \Delta \rho_{\mathrm{Ga}-\mathrm{H}}$; the magnetization $\mu$; the cohesive energy per atom, $E_{\mathrm{Coh} / \text { atom; }}$ and work function, $\Phi$. The in-plane stiffness, $C_{\mathrm{a}}$ and $C_{\mathrm{b}}$, and Poisson ratio, $\nu_{\mathrm{a}}$ and $\nu_{\mathrm{b}}$.

Surface functionalization is an alternative approach to strain that can improve the stability of $2 \mathrm{D}$ materials and for tuning their structural, ${ }^{31,32}$ electronic, ${ }^{33,34}$ and magnetic ${ }^{35}$ properties. Generally, hydrogenation is utilized experimentally for surface functionalization because it is a simple and versatile method. ${ }^{36}$ Here, motivated by a recent study that revealed highly anisotropic, partially filled Dirac cones and nonlinear dispersive bands in w-gallenene, we present a comprehensive investigation of the surface functionalization of $\mathrm{w}$-gallenene via oneand two-sided hydrogenation. The effect of hydrogenation on the structural, phononic, electronic, and mechanical properties of w-gallenene are investigated in detail.

The paper is organized as follows. The computational methodology is given in Section 2. The structural, vibrational, and electronic properties of pristine w-gallenene are discussed in Section 3.1. The adsorption of a single $\mathrm{H}$ atom and $\mathrm{H}_{2}$ molecule on w-gallenene are presented in Section 3.2.1. In Sections 3.2.2 and 3.2.3, the structural, phononic and electronic properties of os- and ts-w-gallenene monolayers are presented, respectively. The mechanical properties of all the monolayers of $\mathrm{w}$-gallenene crystals as their linear-elastic constants, in-plane stiffness and Poisson ratio, are discussed in Section 3.3. We conclude our results in Section 4.

\section{COMPUTATIONAL DETAILS}

First-principles calculations were performed within the density functional theory formalism by using the Vienna $a b$ initio simulation package. ${ }^{37}$ To approximate the exchange and correlation energies, Perdew-Burke-Ernzerhof form of the generalized gradient approximation (GGA) functional was used. $^{38}$ The analysis of the charge transfers between individual atoms was done by the Bader technique. ${ }^{39}$

The kinetic energy cutoff for plane-wave expansion was set to $500 \mathrm{eV}$ and the energy was minimized until its variation in the following steps became less than $10^{-8} \mathrm{eV}$, and the Hellmann-Feynman forces on each unit cell was taken to be less than $10^{-7} \mathrm{eV} / \AA \AA$. A $19 \times 19 \times 1 \Gamma$-centered $k$-point sampling was used for the primitive unit cell for band structure calculations performed within spin-orbit coupling (SOC). The metallicity of the monolayers of gallenene and its derivatives is also examined by performing electronic band structure calculations including local density approximation (LDA), Heyd-Scuseria-Ernzerhof (HSE), and meta-GGA functionals (see Figure $S 1$ in the Supporting Information (SI)). The Methfessel-Paxton scheme broadening for the density of states calculation was taken to be $0.2 \mathrm{eV}$. To avoid interaction between adjacent layers, a vacuum space of $\sim 15 \AA$ was implemented. The phonon dispersions were calculated by using the PHON code, ${ }^{40}$ which uses the force constants calculated with the small-displacement method. The cohesive energy per atom $E_{\mathrm{Coh}}$ was calculated using

$$
E_{\mathrm{Coh}}=\frac{1}{n_{\mathrm{tot}}}\left[\sum_{i=1} n_{i} E_{i}-E_{\mathrm{sl}}\right]
$$

where $E_{i}$ represents the energies of a single isolated atom and the $i$ index denotes the type of atom. $E_{\mathrm{sl}}$ represents the total ground-state energy of the related monolayer structures and $n_{\text {tot }}$ and $n_{i}$ are the total number of atoms and the number of isolated $i$ type atoms within the computational unit cell, respectively. The binding energy of an adsorbed atom and molecule was calculated as

$$
E_{\mathrm{b}}=E_{\mathrm{sl}}+E_{\mathrm{ad}}-E_{\mathrm{sl}+\mathrm{ad}}
$$

where $E_{\mathrm{sl}}$ denotes the energy of the monolayer, $E_{\mathrm{ad}}$ is the energy of an isolated adatom or admolecule, and $E_{\text {sl+ad }}$ denotes the total energy when the adatom or admolecule is adsorbed on the monolayer. In addition, the thermal stability of all the monolayer gallenene crystals was examined by performing $a b$ initio molecular dynamics (MD) calculations (see Figure S2 in the SI). 


\section{RESULTS}

\subsection{Washboard Structure of Single-Layer Gallenene.} In this section, the structural, electronic, and vibrational properties of w-gallenene, a single plane of bulk $\alpha$-Ga exfoliated along the (010) direction, are investigated. This monolayer is the most suitable gallenene phase for hydrogenation and is similar to the anisotropic structure of wborophene. ${ }^{17}$ The optimized atomic structure of w-gallenene resembles a zigzag orthorhombic structure, which is like a washboard structure. As shown in Figure 1a, it forms a 2D double-decker structure, where the two Ga atoms are separated by $t=1.27 \AA$ along the out-of-plane direction, which is defined as the buckling height. The primitive unit cell of the structure includes four Ga atoms. The lattice constants of the primitive unit cell are calculated to be $a=4.65 \AA$ and $b=4.82 \AA$. The $\mathrm{Ga}-\mathrm{Ga}$ bond lengths are found to be $\delta_{1}=2.71 \AA$ and $\delta_{2}=2.73$ $\AA$. It is revealed that the nonmagnetic ground state of wgallenene is formed with a cohesive energy of $2.32 \mathrm{eV} /$ atom. In addition, as given in Table 1 , the work function of the monolayer w-gallenene is $\sim 4.03 \mathrm{eV}^{41}$

For a reliable analysis of the dynamical stability of the wgallenene sheet, it is required to examine the phonon band structure in the whole BZ. The unit cell of w-gallenene consists of 4 atoms, which yields 12 phonon branches, 3 of which are acoustic (longitudinal acoustic, transverse acoustic, and out-ofplane flexural), whereas the other 9 are optical phonon branches. The force constant matrix is calculated by displacing the atoms from their equilibrium positions in a $5 \times 5 \times 1$ supercell. It is found that w-gallenene is dynamically unstable because its phonon dispersion displays imaginary frequencies between the $\Gamma$ and $Y$ high-symmetry points, as shown in Figure $1 \mathrm{~b}$. However, the instability of monolayer w-gallenene is suppressed by applying $2 \%$ of biaxial strain, which can be realized by an appropriate substrate. Furthermore, due to atomic mass and bonding type of Ga atoms, the phonon modes of w-gallenene lie at much lower energies when compared to those of the other $2 \mathrm{D}$ materials such as graphene $\left(\sim 1500 \mathrm{~cm}^{-1}\right)$, silicene $\left(\sim 580 \mathrm{~cm}^{-1}\right)$, and h-BN $(\sim 1600$ $\left.\mathrm{cm}^{-1}\right) .42-44$

The electronic properties of $\mathrm{w}$-gallenene are investigated in terms of its electronic band structure and corresponding orbital projected partial density of states (PDOS), as shown in Figure $1 \mathrm{c}$. It is found that w-gallenene possesses metallic character. Unlike other 2D monoatomic materials, the bands are highly dispersive, with a large bandwidth and increased anisotropy in the low-energy bands. Therefore, w-gallenene exhibits lighter charge carriers and higher mobilities. In addition, it is seen in Figure 1c that the SOC has a negligibly small effect on the electronic band structure. Moreover, PDOS shows that the conduction band (CB) and valence band (VB) states are mainly made up of the p-orbitals of the $\mathrm{Ga}$ atoms.

3.2. Hydrogenation of w-Gallenene. Monolayer wgallenene was successfully synthesized on $\mathrm{SiO}_{2}$ substrate and can be dynamically stabilized upon application of a biaxial strain, ${ }^{30}$ which can be realized by an appropriate substrate. A question naturally arises whether the dynamical instability of free-standing w-gallenene can be suppressed only by biaxial strain or is there any other possible methodology to stabilize the monolayer. Therefore, we address the aforementioned question by studying the effect of surface functionalization with $\mathrm{H}$ atoms as an effective way to enhance the dynamical stability in monolayer w-gallenene.
3.2.1. Interaction of Single $\mathrm{H}$ Atom and $\mathrm{H}_{2}$ Molecule with $w$-Gallenene. Understanding the adsorption properties of a single $\mathrm{H}$ atom and $\mathrm{H}_{2}$ molecule on w-gallenene is important to understand its hydrogenated derivatives. As seen in Figure 2b,

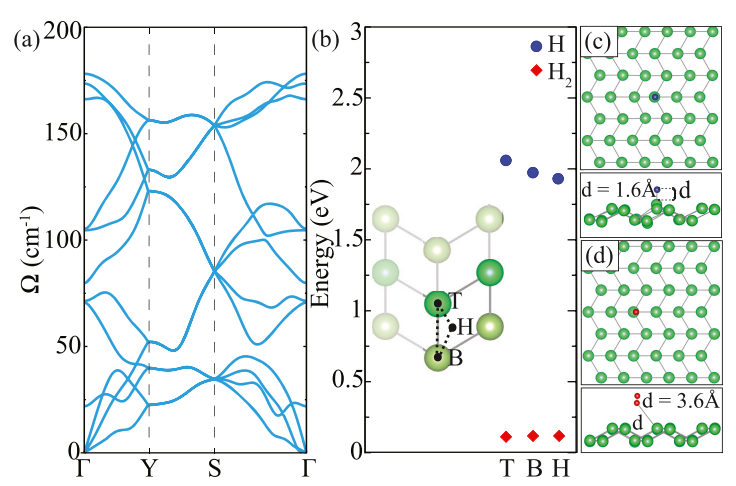

Figure 2. (a) Phonon dispersion of $2 \%$ uniformly strained $\mathrm{w}$ gallenene. (b) The binding energies of the single $\mathrm{H}$ adatom and $\mathrm{H}_{2}$ admolecule on $2 \%$ uniformly strained $\mathrm{w}$-gallenene surface. The inset shows the possible hydrogenation pathways on the w-gallenene surface. The upper Ga atoms are denoted by dark green, whereas the lower ones are denoted by light green. Top and side views of the energetically most favorable adsorption sites of (c) $\mathrm{H}$ atom and (d) single $\mathrm{H}_{2}$ molecule on $3 \times 3 \times 1$ super cell w-gallenene.

$\mathrm{w}$-gallenene is dynamically stable under $2 \%$ biaxial strain. Therefore, the adsorption of a single $\mathrm{H}$ atom and $\mathrm{H}_{2}$ molecule on $2 \%$ biaixally strained w-gallenene are simulated by considering three inequivalent initial adsorption sites: top of lower $\mathrm{Ga}$ atom (B), top of upper $\mathrm{Ga}$ atom $(\mathrm{T})$, and hollow (H) site, which allows more freedom for taking a position between $\mathrm{T}$ and $\mathrm{H}$, as shown in the inset of Figure $2 \mathrm{a}$.

To eliminate $\mathrm{H}-\mathrm{H}$ and $\mathrm{H}_{2}-\mathrm{H}_{2}$ interactions, the size of the supercell is chosen to be $3 \times 3 \times 1$. In the case of the $\mathrm{H}$ adatom, the bond length of $\mathrm{Ga}-\mathrm{H}$ is $\sim 1.61 \AA$, which is close to the electron-diffraction measurement result $(\sim 1.52 \AA)$ of $\mathrm{Ga}_{2} \mathrm{H}_{6}{ }^{45}$ However, in the case of the $\mathrm{H}_{2}$ admolecule, the distance between $\mathrm{Ga}$ and the molecule is calculated to be $\sim 3.55 \AA$. The highest binding energy of $\mathrm{H}$ adatom is found to be $\sim 2.21 \mathrm{eV}$, when it is adsorbed on the lower $\mathrm{Ga}$ atom. Nevertheless, the highest binding energy of the $\mathrm{H}_{2}$ admolecule is found to be $\sim 0.29 \mathrm{eV}$ for the case in which $\mathrm{H}_{2}$ resides on the upper $\mathrm{Ga}$ atom. The presence of the w-gallenene surface lowers the $\mathrm{H}_{2}$ molecule adsorption barrier and allows for the adsorption reaction at energies well below the dissociation threshold of the $\mathrm{H}-\mathrm{H}$ bond. The $\mathrm{H}_{2}$ molecule should contain considerable binding energy to be chemically adsorbed by the $\mathrm{w}$-gallenene surface. However, the binding energy is quite low and, thus, $\mathrm{H}_{2}$ is physisorbed on the w-gallenene surface.

As shown in Figure $2 \mathrm{c}, \mathrm{d}$, the local atomic structure is negligibly reconstructed by the adsorbed $\mathrm{H}$ atom and $\mathrm{H}_{2}$ molecule. On the basis of the Bader charge analysis, it is found that $\mathrm{Ga}$ atom donates $0.3 \mathrm{e}^{-}$to the bonding $\mathrm{H}$ atom, which reveals the partial ionic bonding between $\mathrm{Ga}$ and $\mathrm{H}$ atoms. However, due to the physisorption of $\mathrm{H}_{2}$ molecule on wgallenene, there is no net charge transfer between the surface and the molecule. Moreover, the nonmagnetic nature of the monolayer remains unchanged with a single $\mathrm{H}$ atom and $\mathrm{H}_{2}$ molecule adsorption. Taking into account the analyses of binding energies, bond length, and charge-transfer results, hydrogenation of w-gallenene is only possible by using atomic hydrogen. 

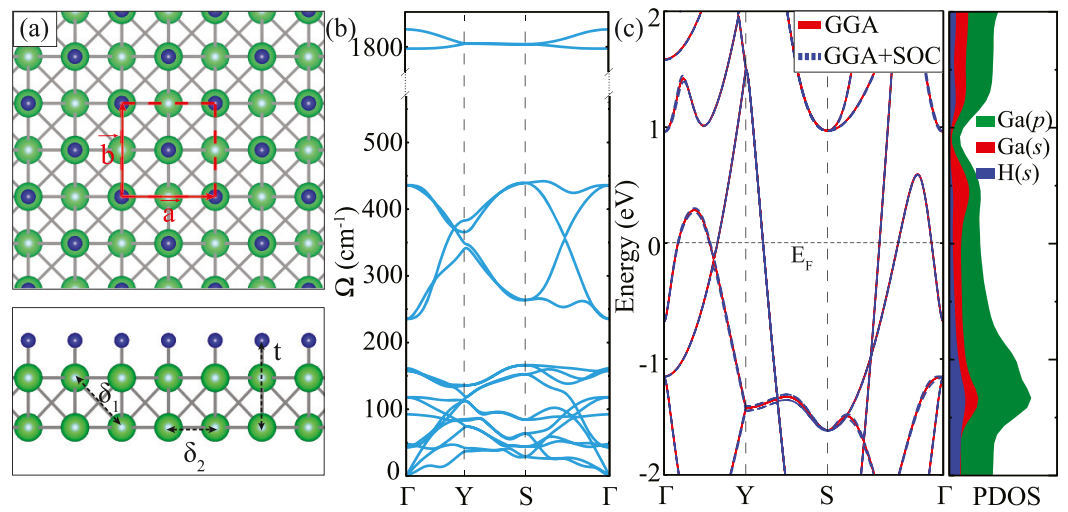

Figure 3. (a) Top and side views of os-w-gallenene. Ga and $\mathrm{H}$ atoms are denoted by green and blue colors, respectively. Red dashed lines represent the rectangular unit cell; $\vec{a}$ and $\vec{b}$ are the lattice vectors. (b) The phonon band structure of os-w-gallenene. (c) The electronic band structure and corresponding orbital projected partial density of states of os-w-gallenene. The Fermi level is set to zero.

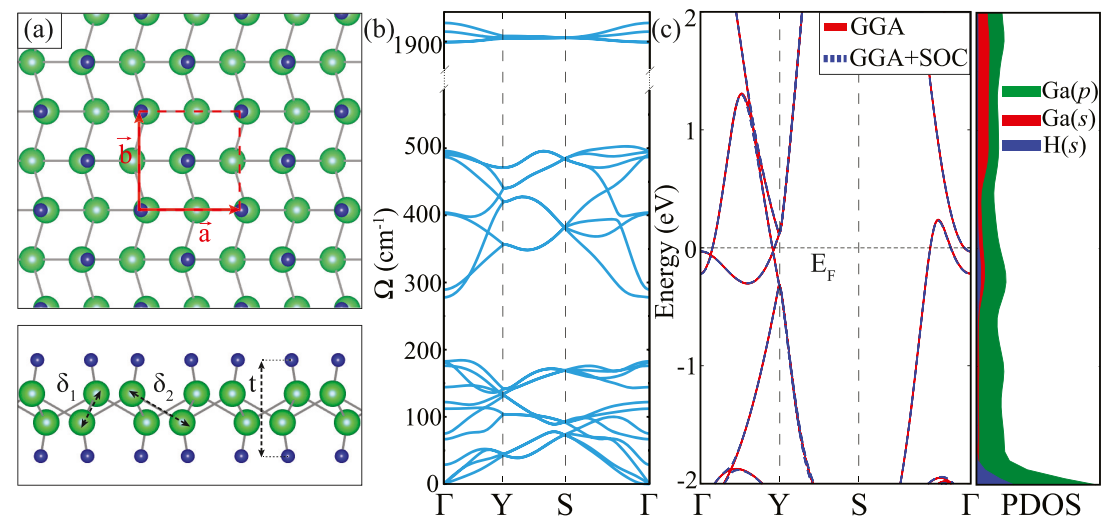

Figure 4. (a) Top and side views of ts-w-gallenene. Ga and $\mathrm{H}$ atoms are denoted by green and blue, respectively. Red dashed lines represent the rectangular unit cell; $\vec{a}$ and $\vec{b}$ are the lattice vectors. (b) The phonon band structure of ts-w-gallenene. (c) The electronic band structure and the corresponding orbital projected partial density of states of ts-w-gallenene. The Fermi level is set to zero.

3.2.2. One-Sided Hydrogenation of $w$-Gallenene. Following the analysis of the adsorption of a single $\mathrm{H}$ atom on $2 \%$ biaxially strained w-gallenene, in this section, the structural, electronic, and phononic properties of one-sided hydrogenated w-gallenene are discussed. To construct the one-side hydrogenated structure, the most favorable position of an isolated $\mathrm{H}$ atom on $2 \%$ biaxially strained w-gallenene is considered. After hydrogenation, the applied strain is removed and the system is found to be dynamically stable without biaxial strain. The crystal structure of os-w-gallenene does not retain the structure of w-gallenene, as shown in Figure 3a. The primitive unit cell of os-w-gallenene includes four $\mathrm{Ga}$ and two $\mathrm{H}$ atoms. The thickness of the monolayer is calculated to be $3.70 \AA$. The lattice constants are $a=b=4.65 \AA$. The $\mathrm{Ga}-\mathrm{Ga}$ bond lengths are found to be 2.82 and $2.90 \AA$ for $\delta_{1}$ and $\delta_{2}$, respectively.

According to Bader charge analysis, Ga atom donates $0.2 \mathrm{e}^{-}$ to $\mathrm{H}$ atom, i.e., $\mathrm{Ga}-\mathrm{H}$ bond possesses a partial ionic character, whereas the covalent bonding character between $\mathrm{Ga}$ atoms is still conserved. The monolayer os- $\mathrm{w}$-gallenene exhibits a nonmagnetic ground state with a cohesive energy of 2.25 $\mathrm{eV} /$ atom, which is slightly $(\sim 0.07 \mathrm{eV})$ lower than that of wgallenene. When $\mathrm{H}$ atoms are adsorbed on the w-gallenene surface, strong $\mathrm{Ga}-\mathrm{H}$ bonds are formed. The work function at the hydrogenated side is increased to $\sim 5.17 \mathrm{eV}$.

The phonon band structure of monolayer os-w-gallenene is shown in Figure 3b. After one-sided hydrogenation, the applied strain is removed and the relaxed system is dynamically stable without a biaxial strain. Notice that the $\mathrm{Ga}-\mathrm{H}$ interaction enhances the dynamical stability of the monolayer w-gallenene. Monolayer os-w-gallenene exhibits 3 acoustic and 15 optical phonon branches. There are three doubly degenerated optical phonon modes having frequencies 43.1, 118.0 , and $157.7 \mathrm{~cm}^{-1}$ at the $\Gamma$ point, which correspond to the in-plane vibration of the atoms. The phonon mode having the frequency $43.1 \mathrm{~cm}^{-1}$ is attributed to the opposite in-plane vibration of lower $\mathrm{Ga}$ with respect to the upper $\mathrm{Ga}$ atoms, whereas the phonon mode with the frequency 118.0 and 157.7 $\mathrm{cm}^{-1}$ are attributed to the opposite in-plane vibration of the Ga atoms in each sublayer. In addition, there are three optical phonon modes having frequencies of 47.8, 92.4, and 161.4 $\mathrm{cm}^{-1}$ at the $\Gamma$ point, which correspond to the out-of-plane vibration of the atoms. The phonon modes with frequency 92.4 and $161.4 \mathrm{~cm}^{-1}$ are attributed to the out-of-plane vibration of one sublayer $\mathrm{Ga}$, whereas the other sublayer Ga atoms are frozen. However, the phonon mode with frequency $47.8 \mathrm{~cm}^{-1}$ is an out-of-plane vibration of lower Ga with respect to the upper Ga atoms. Furthermore, owing to the smaller atomic radius of the $\mathrm{H}$ atom and the relatively strong chemical bonding in os-w-gallenene, the phonon frequencies harden. The modes with higher frequencies occur between 1800 and $1850 \mathrm{~cm}^{-1}$ (see Figure 3b), which have only out-of-plane character.

As shown in Figure $3 c$, the monolayer os-w-gallenene possesses the characteristic properties of metal with nonzero 
density of states at the Fermi level. The calculated PDOS reveals that both $\mathrm{CB}$ and $\mathrm{VB}$ states are mainly made up of the p- and the s-orbitals of the $\mathrm{Ga}$ atom and s-orbital of $\mathrm{H}$ atom. The electronic band structure of os-w-gallenene is highly dispersive, with a large bandwidth in the vicinity of the Fermi level; therefore, it possesses a high charge carrier mobility. The breaking of spin degeneracy at the $\mathrm{CB}$ and VB edges of os-wgallenene stems from the edge asymmetry of its structure, in strict contrast to w-gallenene.

3.2.3. Two-Sided Hydrogenation of $w$-Gallenene. In this section, the structural, electronic, and phononic properties of ts-w-gallenene are discussed in detail. In the case of the unstrained ground state, it is found that the crystal structure of ts-w-gallenene is almost the same as that of w-gallenene, as shown in Figure 4a. The primitive unit cell is marked by a red dashed rectangle, which contains four $\mathrm{Ga}$ and four $\mathrm{H}$ atoms. Each $\mathrm{Ga}$ atom is saturated with a $\mathrm{H}$ atom at the top site. Similar to the case of w-gallenene, it has a buckled structure, with a buckling height of $4.44 \AA$ \& between the uppermost and the lowermost $\mathrm{H}$ atoms. The ts-w-gallenene has a zigzag orthorhombic structure with lattice constants $a=4.62 \AA$ and $b$ $=4.55 \AA$. The $\mathrm{Ga}-\mathrm{Ga}$ bond lengths are found to be 2.72 and $2.66 \AA$ for $\delta_{1}$ and $\delta_{2}$, respectively.

The Bader charge analysis reveals that each $\mathrm{Ga}$ atom donates $0.3 \mathrm{e}^{-}$to each $\mathrm{H}$ atom, which indicates that the $\mathrm{Ga}-\mathrm{H}$ bond possesses a partially ionic character. It is seen that the hydrogenation leads to increase in ionic character of the bonds between $\mathrm{Ga}$ and $\mathrm{H}$ atoms, whereas the covalency of the $\mathrm{Ga}-$ $\mathrm{Ga}$ bond is still conserved because there occurs no charge transfer between $\mathrm{Ga}$ atoms. Similar to the cases of w-gallenene and os-w-gallenene, the ground-state structure of the ts-wgallenene is also found to be nonmagnetic. It is found that the cohesive energy of ts-w-gallenene is the same as that of os-wgallenene. The resulting two-sided hydrogenation of wgallenene leads to the formation of strong $\mathrm{Ga}-\mathrm{H}$ bonds. Hence, ts-w-gallenene has a work function that is $\sim 1.2 \mathrm{eV}$ higher than that of w-gallenene.

The phonon dispersion of ts-w-gallenene is shown in Figure 4b. Similar to the case of os-w-gallenene, the two-sided hydrogenation of $\mathrm{w}$-gallenene suppresses its dynamical instability when the biaxial strain is removed. It exhibits 3 acoustic and 21 optical phonon branches. Two acoustical phonon branches of ts-w-gallenene are linear as $k \rightarrow 0$, but its transverse branch displays a quadratic dispersion near the $\Gamma$ point.

Among the optical vibrational modes, the phonon modes having frequencies 28.8, 66.7, 176.0, and $182.6 \mathrm{~cm}^{-1}$ are attributed to the opposite in-plane vibration of $\mathrm{Ga}$ with respect to the closest $\mathrm{Ga}$ atom. The phonon modes with the frequencies 75.1 and $144.2 \mathrm{~cm}^{-1}$ are due to the opposite inplane vibration of the lower $\mathrm{Ga}$ with respect to the upper $\mathrm{Ga}$ atoms. The phonon modes at frequencies 112.1 and 121.9 $\mathrm{cm}^{-1}$ are attributed to the opposite out-of-plane vibration of $\mathrm{Ga}$ with respect to the closest $\mathrm{Ga}$ atom. Furthermore, the phonon mode having frequency $180.9 \mathrm{~cm}^{-1}$ reveals the opposite out-of-plane vibration of the lower $\mathrm{Ga}$ with respect to the upper Ga atoms. Similar to the case of os-w-gallenene, the $\mathrm{Ga}-\mathrm{H}$ bonds have much higher frequencies than the $\mathrm{Ga}-$ $\mathrm{Ga}$ bonds, as shown in Figure $4 \mathrm{~b}$. The higher frequencies of out-of-plane modes between 1940 and $2000 \mathrm{~cm}^{-1}$ indicate the formation of strong $\mathrm{Ga}-\mathrm{H}$ bonds as well.

As shown in Figure 4c, ts-w-gallenene also possesses metallic character because of the finite density of states at the Fermi energy. It is found from the PDOS calculation that the $\mathrm{CB}$ states are mainly made up of the $\mathrm{p}$ - and s-orbitals of the $\mathrm{Ga}$ atoms, whereas the states in the VB have the character of $\mathrm{p}$ orbital of $\mathrm{Ga}$ atom and s-orbital of $\mathrm{H}$ atom. Unlike os-wgallenene, there is a notable change in the dispersion in the vicinity of the Fermi level. The bands of w-gallenene only cross at the Fermi level between the $Y$ and $\Gamma$ high symmetry points. As in the case of w-gallenene, negligibly small spin-orbit splittings occur in the low-energy valence bands. Furthermore, similar to the cases of $\mathrm{w}$-gallenene and os-w-gallenene, the electronic bands of w-gallenene are highly dispersive, with a large bandwidth in the vicinity of the Fermi level, which indicates a high charge carrier mobility.

3.3. Mechanical Properties of w-Gallenene, osGallenene, and ts-w-Gallenene. The mechanical properties of the monolayers, w-gallenene, os-gallenene, and ts-wgallenene, are discussed in terms of their linear-elastic parameters. The linear-elastic properties of two-dimensional homogeneous monolayer materials can be represented by two independent constants, the in-plane stiffness, $C$, and the Poisson ratio, $\nu$.

The linear-elastic constants of the monolayers, w-gallenene, os-gallenene, and ts-w-gallenene, are determined by using the energy-strain relation. Super cells with 64, 96, and 128 atoms are constructed for bare, os-gallenene, and ts-w-gallenene crystals, respectively. The tensile and compressive strains are applied along the $a$ and $b$ vectors, respectively. The strain parameters $\varepsilon_{\mathrm{a}}$ and $\varepsilon_{\mathrm{b}}$ are varied between \pm 0.015 , with a step size of 0.005 . Then, three different sets of data are calculated: (i) $\varepsilon_{\mathrm{a}}=0$ and $\varepsilon_{\mathrm{b}}$ varying, (ii) $\varepsilon_{\mathrm{b}}=0$ and $\varepsilon_{\mathrm{a}}$ varying, and (iii) $\varepsilon_{\mathrm{a}}$ $=\varepsilon_{\mathrm{b}}$ varying. At each configuration, the atomic positions are fully relaxed and the strain energy, $E_{S}$, is calculated by subtracting the total energy of the equilibrium state from the strained structure. Then, the calculated data are fitted to the equation: $E_{\mathrm{S}}=c_{1} \varepsilon_{\mathrm{a}}{ }^{2}+c_{2} \varepsilon_{\mathrm{b}}{ }^{2}+c_{3} \varepsilon_{\mathrm{a}} \varepsilon_{\mathrm{b}}{ }^{46}$ and the coefficients $c_{i}$ are determined.

The in-plane stiffness, $C$, is a measure of the rigidity of a material under applied load. For a $2 \mathrm{D}$ crystal, the larger $C$ demonstrates less stretchability of the material. In isotropic monolayers, no orientational dependence in the crystal is expected. However, if there is even a small anisotropy in the material, the stiffness along the two lattice vectors is given by $C_{\mathrm{a}}=\left(1 / A_{0}\right)\left(2 c_{1}-c_{3}{ }^{2} / 2 c_{2}\right)^{47}$ and $C_{\mathrm{b}}=\left(1 / A_{0}\right)\left(2 c_{2}-c_{3}{ }^{2} /\right.$ $\left.2 c_{1}\right),{ }^{47}$ where $c_{1} \neq c_{2}$ due to the anisotropy of the crystal structure and $A_{0}$ is the area of the unstrained super cell. The rigidity of the monolayer w-gallenene may be clearly understood by comparing its in-plane stiffness with that of other monoatomic $2 \mathrm{D}$ materials. We find $C_{\mathrm{a}} \approx C_{\mathrm{b}} \approx 25 \mathrm{~N} / \mathrm{m}$ (see Table 1), which is practically isotropic. When compared with that of graphene $\left(330 \mathrm{~N} / \mathrm{m}^{48}\right)$, monolayer w-gallenene has a very small in-plane stiffness, which is a consequence of the relatively weak $\mathrm{Ga}-\mathrm{Ga}$ bonds in the crystal. For monoatomic crystals of $\mathrm{Si}$ and $\mathrm{Ge}$, respectively, the stiffnesses of 62 and $48 \mathrm{~N} / \mathrm{m}$ were reported, ${ }^{48}$ which are even higher than those of w-gallenene. Very low in-plane stiffness of w-gallenene reveals that it withstands high values of strain at low stresses.

In the case of os-w-gallenene, the presence of $\mathrm{H}$ atoms results in an increase in the stiffness, and the calculated stiffnesses are $C_{\mathrm{a}} \approx C_{\mathrm{b}} \approx 52 \mathrm{~N} / \mathrm{m}$, indicating the isotropic nature of the crystal structure. Increased in-plane stiffness expresses itself in the higher phonon frequencies, as shown in Figure $3 \mathrm{~b}$. The same trend is found for the monolayer ts-wgallenene, whose in-plane stiffnesses, $C_{\mathrm{a}}=48 \mathrm{~N} / \mathrm{m}$ and $C_{\mathrm{b}}=47$ 

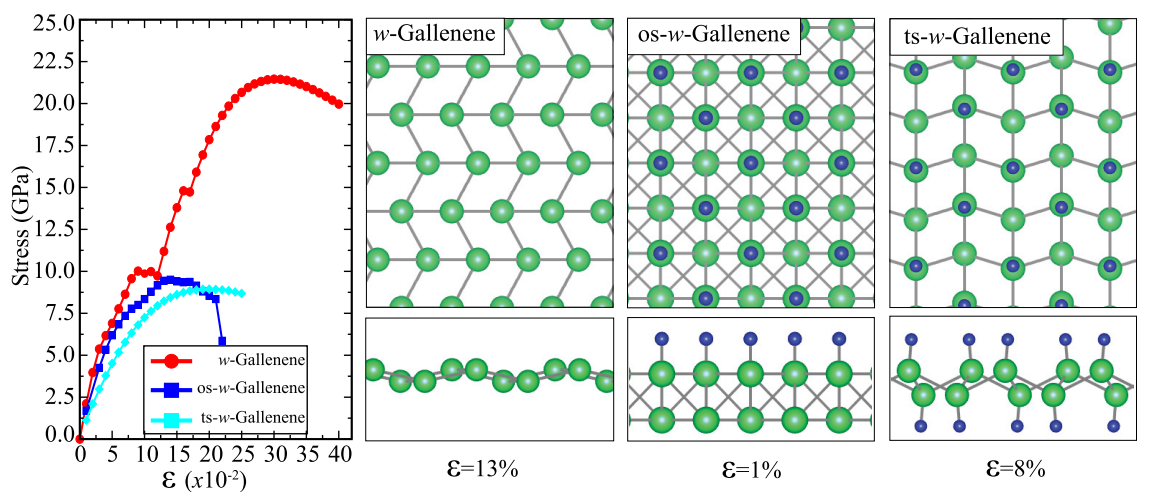

Figure 5. Left panel shows the stress-strain energy curves under applied biaxial strain for three monolayers. Right panel, the crystal structures of the three different monolayers are shown at the maximum biaxial strains, after which the phononic instabilities occur.

$\mathrm{N} / \mathrm{m}$, are slightly different along the two directions. In contrast to other hydrogenated monolayer crystals, graphane ${ }^{49-51}$ and $1 \mathrm{~T}-\mathrm{ReS}_{2} \mathrm{H}_{2}$, ${ }^{31}$ which soften when hydrogenated, in the case of w-gallenene, surface hydrogenation forms relatively strong $\mathrm{Ga}-\mathrm{H}$ bonds, which tunes the $\mathrm{Ga}-\mathrm{Ga}$ bonds to result in an increased in-plane stiffness.

The Poisson ratio of a material is defined as the ratio of the transverse contraction strain to the longitudinal extension strain in the direction of the stretching force, i.e., $\nu=\varepsilon_{\text {trans }}$ / $\varepsilon_{\text {long }}$. The Poisson ratio for $a$ - and $b$-vectors can be calculated in terms of the coefficients $c_{i}$ as $\nu_{\mathrm{a}}=c_{3} / 2 c_{2}$ and $c_{3} / 2 c_{1}$. The Poisson ratios for w-gallenene are $\nu_{\mathrm{a}} \approx \nu_{\mathrm{b}} \approx 0.20$. These values are smaller as compared to those for the well-known TMDs, i.e., 0.25 for $\mathrm{MoS}_{2},{ }^{52} 0.22$ for $\mathrm{WS}_{2},{ }^{5252}$ and 0.23 for $\mathrm{MoSe}_{2}{ }^{52}$ The Poisson ratio displays different behavior depending on the type of hydrogenation in the case of w-gallenene. For the monolayer os-w-gallenene, it is 0.26 , which is larger than that of graphene (0.19). ${ }^{48}$ This indicates a larger extension of the crystal in the transverse direction, when it is contracted along the longitudinal direction. However, when both surfaces are hydrogenated, the Poisson ratio is $\nu_{\mathrm{a}} \approx \nu_{\mathrm{b}} \approx 0.25$.

The ultimate strength of a material is the maximum value of stress that a material can resist before fracturing. This value can be directly investigated from the maximum point of the stressstrain curve of a material. To investigate the nonlinear-elastic properties of the gallium monolayers, the large biaxial strain is applied to the crystals. As seen on the left panel of Figure 5, monolayers of w-gallenene and its derivatives do not exhibit a fracture even at large biaxial strain. The ultimate strength of the monolayer w-gallenene is calculated to be $22 \mathrm{GPa}$, which is much lower than that of graphene $\left(96 \mathrm{GPa}^{28}\right)$ but close to that of monolayer $\mathrm{MoS}_{2}\left(26 \mathrm{GPa}^{28}\right)$ and even much higher than that of Ga-monochalcogenides (12 and $10 \mathrm{GPa}$ for GaS and $\mathrm{GaSe}$, respectively). ${ }^{28}$ In addition, the ultimate strain value, i.e., the strain at which the material exhibits its ultimate strength, is higher ( $30 \%$ for monolayer w-gallenene) than that for Gamonochalcogenides (24 and $23 \%$ for $\mathrm{GaS}$ and GaSe, respectively). ${ }^{28}$ Furthermore, we analyze the phonon instability under applied biaxial strain. As shown in the right panel of Figure 5, the maximum biaxial strains are 13,1 , and $8 \%$ for wgallenene, os-w-gallenene, and ts-w-gallenene, respectively; for which the phonon instabilities occur. Especially, the phonon stabilities of w-gallenene against biaxial tensile strain values are higher than those for the superstretchable borophene $(8 \%){ }^{53}$

In contrast to the results for the linear-elastic parameters, hydrogenation of w-gallenene decreases both the ultimate strength and the strain values. In the case of one-sided hydrogenation, the material withstands a maximum load of 12 $\mathrm{GPa}$ at a relatively much lower strain value of $14 \%$. After monolayer os-w-gallenene exhibits its maximum stress at $14 \%$ of biaxial strain, it does not show a crystal fracture up to a strain value of $22 \%$, at which value there is a sharp decrease in the stress (see Figure 5). However, the phonon instability occurs just at $1 \%$ of biaxial strain, which suggests that onesided hydrogenation is probably not feasible. In the case of monolayer ts-w-gallenene, the ultimate strength shows a decrease in the stress to $9 \mathrm{GPa}$, but at a larger strain value of $20 \%$. This larger ultimate strain can be related to the distorted $\mathrm{Ga}-\mathrm{H}$ bonds at both surfaces, which are more flexible than those in the monolayer os-w-gallenene. As seen in Figure 5, the phonon instability occurs at $8 \%$ of the biaxial strain, which is the same as that of borophene itself $(8 \%){ }^{53}$

To characterize the corrugation of $2 \mathrm{D}$ materials, the buckling height, $t$, is an important parameter. ${ }^{54,55}$ The buckling height decreases monotonously with increasing strain before the strain approaches the critical value (12\%), but it quickly decreases to zero as the strain continuously increases. Until applying $8 \%$ biaxial tensile strain, w-gallenene possesses more superior flexibility and isotropic character because it perceives approximately the same in-plane $a$ and $b$ directional stress. However, there is different $a$ and $b$ directional tension between applying 8 and $12 \%$ tensile strain, so the stress-dependent buckling height is nonmonotonic. Between the stresses of 12 and $17 \%$, the structure is elongated differently in the $x$ - and $y$ directions, which increases its anisotropy. Hence, w-gallenene turns into a graphene-like planar structure instead of the original bucking structure in this case.

\section{CONCLUSIONS}

The structural, phononic, electronic, and mechanical properties of pristine and hydrogenated monolayers of w-gallenene were investigated within the state-of-the-art first-principles calculations. We showed that a single $\mathrm{H}$ atom can be adsorbed to the surface of w-gallenene with a high binding energy (2.21 $\mathrm{eV})$. One- and two-sided hydrogenated w-gallenene are dynamically stable. Electronic structure analyses revealed that all monolayers, w-gallenene, os-w-gallenene, and ts-w-gallenene, are nonmagnetic metals. Moreover, the linear-elastic constants, in-plane stiffness and Poisson ratio, of the monolayer w-gallenene are enhanced by one- and two-sided hydrogenation, which is the opposite trend found for other hydrogenated monolayer crystals. However, in the nonlinearelastic regime, it was found that the ultimate strength and the 
ultimate strain decrease as the number of hydrogenated surfaces increase. Furthermore, the phonon stabilities of monolayer $\mathrm{w}$-gallenene and ts-w-gallenene remain up to relatively higher biaxial strains when compared to borophene. With its enhanced dynamical stability, robust metallic character, and enhanced linear-elastic properties, hydrogenated monolayer w-gallenene is a potential candidate for nanodevice applications as a $2 \mathrm{D}$ flexible metal.

\section{ASSOCIATED CONTENT}

\section{S Supporting Information}

The Supporting Information is available free of charge on the ACS Publications website at DOI: 10.1021/acs.jpcc.8b07353.

Electronic band structures of $\mathrm{w}$-gallenene, os-gallenene, and ts-w-gallenene by using GGA, LDA, GGA + HSE, and meta-GGA functionals (Figure S1); thermal stability of the all monolayer gallenene crystals (Figure S2) (PDF)

\section{AUTHOR INFORMATION}

\section{Corresponding Authors}

*E-mail: sabuhibadalov@iyte.edu.tr (S.V.B.).

*E-mail: Mehmet.Yagmurcukardes@uantwerpen.be (M.Y.).

\section{ORCID $\odot$}

M. Yagmurcukardes: 0000-0002-1416-7990

H. Sahin: 0000-0002-6189-6707

\section{Notes}

The authors declare no competing financial interest.

\section{ACKNOWLEDGMENTS}

Computational resources were provided by TUBITAK ULAKBIM, High Performance and Grid Computing Center (TR-Grid e-Infrastructure). H.S. acknowledges support from Turkish Academy of Sciences under the GEBIP program. This work was supported by FLAG-ERA project TRANS-2D-TMD. This work is supported by the Flemish Science Foundation (FWO-Vl) by a postdoctoral fellowship (M.Y.).

\section{REFERENCES}

(1) Novoselov, K. S.; Geim, A. K.; Morozov, S. V.; Jiang, D.; Zhang, Y.; Dubonos, S. V.; Grigorieva, I. V.; Firsov, A. A. Electric Field Effect in Atomically Thin Carbon Films. Science 2004, 306, 666-669.

(2) Geim, A. K.; Novoselov, K. S. The Rise of Graphene. Nat. Mater. 2007, 6, 183-191.

(3) Kim, K. K.; Hsu, A.; Jia, X.; Kim, S. M.; Shi, Y.; Hofmann, M.; Nezich, D.; Rodriguez-Nieva, J. F.; Dresselhaus, M.; Palacios, T.; Kong, J. Synthesis of Monolayer Hexagonal Boron Nitride on Cu Foil Using Chemical Vapor Deposition. Nano Lett. 2012, 12, 161-166.

(4) Manzeli, S.; Ovchinnikov, D.; Pasquier, D.; Yazyev, O. V.; Kis, A. 2D transition metal dichalcogenides. Nat. Rev. Mater. 2017, 2, No. 17033.

(5) Coleman, J. N.; Lotya, M.; O’Neill, A.; Bergin, S. D.; King, P. J.; Khan, U.; Young, K.; Gaucher, A.; De, S.; Smith, R. J.; et al. Twodimensional nanosheets produced by liquid exfoliation of layered materials. Science 2011, 331, 568-571.

(6) Chhowalla, M.; Shin, H. S.; Eda, G.; Li, L. J.; Loh, K. P.; Zhang, $\mathrm{H}$. The chemistry of two-dimensional layered transition metal dichalcogenide nanosheets. Nat. Chem. 2013, 5, 263-275.

(7) Kara, A.; Enriquez, H.; Seitsonen, A. P.; Voon, L. C. L. Y.; Vizzini, S.; Aufray, B.; Oughaddou, H. A review on silicene-new candidate for electronics. Surf. Sci. Rep. 2012, 67, 1-18.

(8) Vogt, P.; Padova, P.; Quaresima, C.; Avila, J.; Frantzeskakis, E.; Asensio, M. C.; Resta, A.; Ealet, B.; Lay, G. L. Silicene: Compelling
Experimental Evidence for Graphenelike Two-Dimensional Silicon. Phys. Rev. Lett. 2012, 108, No. 155501.

(9) De Padova, P.; Kubo, O.; Olivieri, B.; Quaresima, C.; Nakayama, T.; Aono, M.; Lay, G. L. Multilayer Silicene Nanoribbons. Nano Lett. 2012, 12, 5500-5503.

(10) Bianco, E.; Butler, S.; Jiang, S.; Restrepo, O. D.; Windl, W.; Goldberger, J. E. Stability and exfoliation of germanane: a germanium graphane analogue. ACS Nano 2013, 7, 4414-4421.

(11) Derivaz, M.; Dentel, D.; Stephan, R.; Hanf, M. C.; Mehdaoui, A.; Sonnet, P.; Pirri, C. Continuous Germanene Layer on $\mathrm{Al}(111)$. Nano Lett. 2015, 15, 2510-2516.

(12) Dávila, M. E.; Xian, L.; Cahangirov, S.; Rubio, A.; Lay, G. L. Germanene: a novel two-dimensional germanium allotrope akin to graphene and silicene. New J. Phys. 2014, 16, No. 095002.

(13) Dávila, M. E.; Lay, G. L. Few layer epitaxial germanene: a novel two-dimensional Dirac material. Sci. Rep. 2016, 6, No. 20714.

(14) Zhu, F.-f.; Chen, W.-j.; Xu, Y.; Gao, C.-1.; Guan, D.-d.; Liu, C.h.; Qian, D.; Zhang, S.-C.; Jia, J.-f Epitaxial Growth of TwoDimensional Stanene. Nat. Mater. 2015, 14, 1020-1025.

(15) Saxena, S.; Chaudhary, R. P.; Shukla, S. Stanene: Atomically Thick Free-standing Layer of 2D Hexagonal Tin. Sci. Rep. 2016, 6, No. 31073.

(16) Li, L.; Yu, Y.; Ye, G. J.; Ge, Q.; Ou, X.; Wu, H.; Feng, D.; Chen, X. H.; Zhang, Y. Black phosphorus field-effect transistors. Nat. Nanotechnol. 2014, 9, 372-377.

(17) Sachdev, H. Disclosing boron's thinnest side. Science 2015, 350, $1468-1469$.

(18) Bernasconi, M.; Chiarotti, G. L.; Tosatti, E. Ab initio calculations of structural and electronic properties of gallium solidstate phases. Phys. Rev. B 1995, 52, 9988-9998.

(19) Kenichi, T.; Kazuaki, K.; Masao, A. High-pressure bct-fcc phase transition in Ga. Phys. Rev. B 1998, 58, 2482-2486.

(20) Bosio, L. Crystal structures of $\mathrm{Ga}(\mathrm{II})$ and $\mathrm{Ga}(\mathrm{III})$. J. Chem. Phys. 1978, 68, 1221-1223.

(21) Steenbergen, K. G.; Gaston, N. First-principles melting of gallium clusters down to nine atoms: structural and electronic contributions to melting. Phys. Chem. Chem. Phys. 2013, 15, 1532515332.

(22) Schulte, O.; Holzapfel, W. Effect of pressure on the atomic volume of $\mathrm{Ga}$ and $\mathrm{Tl}$ up to $68 \mathrm{GPa}$. Phys. Rev. B 1997, 55, 81228128.

(23) Hall, R. N.; Fenner, G. E.; Kingsley, J. D.; Soltys, T. J.; Carlson, R. O. Coherent Light Emission From GaAs Junctions. Phys. Rev. Lett. 1962, 9, 366-369.

(24) Amano, H.; Sawaki, N.; Akasaki, I.; Toyoda, Y. Metalorganic vapor phase epitaxial growth of a high quality GaN film using an AlN buffer layer. Appl. Phys. Lett. 1986, 48, 353-355.

(25) Zhang, H.-M.; Sun, Y.; Li, W.; Peng, J.-P.; Song, C.-L.; Xing, Y.; Zhang, Q.; Guan, J.; Li, Z.; Zhao, Y.; et al. Detection of a Superconducting Phase in a Two-Atom Layer of Hexagonal Ga Film Grown on Semiconducting GaN(0001). Phys. Rev. Lett. 2015, 114, No. 107003.

(26) Zhou, X.; Cheng, J.; Zhou, Y.; Cao, T.; Hong, H.; Liao, Z.; Wu, S.; Peng, H.; Liu, K.; Yu, D. Strong Second-Harmonic Generation in Atomic Layered GaSe. J. Am. Chem. Soc. 2015, 137, 7994-7997.

(27) Carey, B. J.; Ou, J. Z.; Clark, R. M.; Berean, K. J.; Zavabeti, A.; Chesman, A. S. R.; Russo, S. P.; Lau, D. W. M.; Xu, Z.-Q.; Bao, Q.; et al. Wafer-scale two-dimensional semiconductors from printed oxide skin of liquid metals. Nat. Commun. 2017, 8, No. 14482.

(28) Yagmurcukardes, M.; Senger, R. T.; Peeters, F. M.; Sahin, H. Mechanical properties of monolayer GaS and GaSe crystals. Phys. Rev. B 2016, 94, No. 245407.

(29) Xing, Y.; Zhang, H.-M.; Fu, H.-L.; Liu, H.; Sun, Y.; Peng, J.-P.; Wang, F.; Lin, X.; Ma, X.-C.; Xue, Q.-K.; et al. Quantum Griffiths singularity of superconductor-metal transition in Ga thin films. Science 2015, 350, 542-545.

(30) Kochat, V.; Samanta, A.; Zhang, Y.; Bhowmick, S.; Manimunda, P.; Asif, S.; Stender, A. S.; Vajtai, R.; Singh, A. K.; et al. Atomically 
thin gallium layers from solid-melt exfoliation. Sci. $A d v$. 2018, 4, No. e1701373.

(31) Yagmurcukardes, M.; Bacaksiz, C.; Senger, R. T.; Sahin, H. Hydrogen-induced structural transition in single layer $\operatorname{ReS}_{2}$. $2 \mathrm{D}$ Mater. 2017, 4, No. 035013.

(32) Li, W.; Kong, L.; Feng, B.; Fu, H.; Li, H.; Zeng, X. C.; Wu, K.; Chen, L. Abnormal phase transition between two-dimensional highdensity liquid crystal and low-density crystalline solid phase. Nat. Commun. 2018, 9, No. 198.

(33) Lei, B.; Pan, Y.; Hu, Z.; Zhang, J.; Xiang, D.; Zheng, Y.; Guo, R.; Han, C.; Wang, L.; Lu, J.; et al. Direct Observation of Semiconductor-Metal Phase Transition in Bilayer Tungsten Diselenide Induced by Potassium Surface Functionalization. ACS Nano 2018, 12, 2070-2077.

(34) Ma, Y.; Liu, B.; Zhang, A.; Chen, L.; Fathi, M.; Shen, C.; Abbas, A. N.; Ge, M.; Mecklenburg, M.; Zhou, C. Reversible Semiconducting-to-Metallic Phase Transition in Chemical Vapor Deposition Grown Monolayer WSe $\mathrm{W}_{2}$ and Applications for Devices. ACS Nano 2015, 9, 7383-7391.

(35) Radhakrishnan, S.; Das, D.; Samanta, A.; de los Reyes, C. A.; Deng, L.; Alemany, L. B.; Weldeghiorghis, T. K.; Khabashesku, V. N.; Kochat, V.; Jin, Z.; et al. Fluorinated h-BN as a magnetic semiconductor. Sci. Adv. 2017, 3, No. e1700842.

(36) Hever, A.; Bernstein, J.; Hod, O. Fluorination Effects on the Structural Stability and Electronic Properties of $\mathrm{sp}^{3}$-type Silicon Nanotubes. J. Phys. Chem. C 2013, 117, 14684-14691.

(37) Kresse, G.; Furthmuller, J. Efficient iterative schemes for $a b$ initio total-energy calculations using a plane-wave basis set. Phys. Rev. B 1996, 54, 11169-11186.

(38) Perdew, J. P.; Burke, K.; Ernzerhof, M. Generalized Gradient Approximation Made Simple. Phys. Rev. Lett. 1996, 77, 3865-3868.

(39) Henkelman, G.; Arnaldsson, A.; Jonsson, H. A fast and robust algorithm for Bader decomposition of charge density. Comput. Mater. Sci. 2006, 36, 354-360.

(40) Alfe, D. PHON: A program to calculate phonons using the small displacement method. Comput. Phys. Commun. 2009, 180, 2622-2633.

(41) Yu, Y. J.; Zhao, Y.; Ryu, S.; Brus, L. E.; Kim, K. S.; Kim, P. Tuning the Graphene Work Function by Electric Field Effect. Nano Lett. 2009, 9, 3430-3434.

(42) Liu, F.; Ming, P.; Li, J. ab initio calculation of ideal strength and phonon instability of graphene under tension. Phys. Rev. B 2007, 76, No. 064120

(43) Cahangirov, S.; Topsakal, M.; Aktürk, E.; Sahin, H.; Ciraci, S. Two- and One-Dimensional Honeycomb Structures of Silicon and Germanium. Phys. Rev. Lett. 2009, 102, No. 236804.

(44) Cassabois, G.; Valvin, P.; Gil, B. Hexagonal boron nitride is an indirect bandgapsemiconductor. Nat. Photonics 2016, 10, 262-266.

(45) Pulham, C. R.; Downs, A. J.; Goode, M. J.; Rankin, D. W. H.; Robertson, H. E. Gallane: synthesis, physical and chemical properties, and structure of the gaseous molecule $\mathrm{Ga}_{2} \mathrm{H}_{6}$ as determined by electron diffraction. J. Am. Chem. Soc. 1991, 113, 5149-5162.

(46) Nye, J. F. Physical Properties of Crystals: Their Representation by Tensors and Matrices; Clarendon Press: Oxford, 1985.

(47) Kang, J.; Sahin, H.; Peeters, F. M. Mechanical properties of monolayer sulphides: a comparative study between $\mathrm{MoS}_{2}, \mathrm{HfS}_{2}$ and $\mathrm{TiS}_{3}$. Phys. Chem. Chem. Phys. 2015, 17, 27742-27749.

(48) Şahin, H.; Cahangirov, S.; Topsakal, M.; Bekaroglu, E.; Aktürk, E.; Senger, R. T.; Ciraci, S. Monolayer honeycomb structures of group-IV elements and III-V binary compounds: First-principles calculations. Phys. Rev. B 2009, 80, No. 155453.

(49) Topsakal, M.; Cahangirov, S.; Ciraci, S. The response of mechanical and electronic properties of graphane to the elastic strain. Appl. Phys. Lett. 2010, 96, No. 091912.

(50) Peelaers, H.; Hernandez-Nieves, A. D.; Leenaerts, O.; Partoens, B.; Peeters, F. M. Vibrational properties of graphene fluoride and graphane. Appl. Phys. Lett. 2011, 98, No. 051914.
(51) Leenaerts, O.; Peelaers, H.; Hernández-Nieves, A. D.; Partoens, B.; Peeters, F. M. First-principles investigation of graphene fluoride and graphane. Phys. Rev. B 2010, 82, No. 195436.

(52) Guzman, D. M.; Strachan, A. Role of strain on electronic and mechanical response of semiconducting transition-metal dichalcogenide monolayers: An ab-initio study. J. Appl. Phys. 2014, 115, No. 243701.

(53) Wang, H.; Li, Q.; Gao, Y.; Miao, F.; Zhou, X. F.; Wan, X. G. Strain effects on borophene: ideal strength, negative Possion's ratio and phonon instability. New J. Phys. 2016, 18, No. 073016.

(54) Wei, Q.; Peng, X. Superior mechanical flexibility of phosphorene and few-layer black phosphorus. Appl. Phys. Lett. 2014, 104, No. 251915.

(55) Hu, T.; Han, Y.; Dong, J. M. Mechanical and electronic properties of monolayer and bilayer phosphorene under uniaxial and isotropic strains. Nanotechnology 2014, 25, No. 455703. 